\title{
The Safety Assessment of the Operation of Urban Rail Transit Based on Generalized Regression Neural Network
}

\author{
Kai Guo ${ }^{1}$, Jingsheng $\mathrm{Gao}^{2}$, Junneng $\mathrm{Ye}^{2}$, Dexin $\mathrm{Yi}^{2}$, Song Kong ${ }^{1}$, Hui Huang ${ }^{1}$, Dan $\mathrm{Qiu}^{1}$, and Wei Gao ${ }^{1 *}$ \\ ${ }^{1}$ Ningbo University of Technology, No. 201 Fenghua Rd, Ningbo, 315211 China \\ ${ }^{2}$ Ningbo Rail Transit Group Co., Ltd., No 3399 Ningchuan Rd, Ningbo, 315101 China
}

\begin{abstract}
This paper presented a methodology to assess the operation safety of the urban rail transit system by using the generalized regression neural network. By comparing the evaluated values with the graded scores of the experts, the results indicated that predictions by using the generalized regression neural network only have $5.38 \%$ averaged relative error compared with experts' scores. Therefore, the GRNN was capable of evaluating and predicting the operation safety of the urban rail transit system.
\end{abstract}

\section{Introduction}

In the modern cities, the traffic problem has become an important issue in the daily life. In order to solve the congestion and improve the overall traffic efficiency, the governments at all levels are vigorously developing public transportation tools. One of them is the urban rail transit system, which is high-speed public transportation with more passengers than the other transportations. With the rapid growth of economy, the constructions of the urban rail transit systems were entering a rapid development period in China. The total mileages of the urban rail transit systems in China have reached 3286 kilometers by the end of 2015 . The total mileage of the urban rail transit system in 2020 is expected to increase $150 \%$ than in 2015 and it will reach 8500 kilometers. Thought the urban rail transit systems have helped to reduce the urban traffic congestion in China, the operation safety becomes an urgent problem since it could cause a huge personnel and/or property damage once the incident happens. For example, on November 11, 1969, three persons died and more than one hundred persons were injured because of the fire accident on the train which was in the interval between Wanshoulu Station and Wukesong Station in Beijing [1]. In November 18, 1987, thirty-one persons were died on the train at Cross Street Station in London due to the fire accident [2]. In February 18, 2003, five hundred and fifty-eight persons were died and two hundred and sixty-nine persons were injured in Daegu's subway in South Korea [3]. Therefore, in order to reduce the fatalities in the accident of the urban rail transit, the operation safety need to be evaluated and predicted.

The methodologies and theories for assessing the operation safety of the urban rail transit have been developed for many years in the past. In London, the safety assessment was used for analyzing the major risk factors and predicting the possible damages for the passengers and staffs with the determination of the quantitative relationship between the damages and the major risk factors [2-3]. In Germany, a professional safety management was performed on vehicles, maintenance, public services, and grid which were appropriate in the safety management. In the United States, the New York Metro transit system has been evaluated for the hazard factors of staffs, passengers, suppliers [4]. The safety assessments reports were issued based on the evaluations, and the regulations in safety management were established. In Beijing, the rules were established to ensure the safety for staffs and passengers.

Among these methodologies, there are many risk factors which affect the operation safety of the urban rail transit. The relationship between the risk factors in the operations of urban rail transit system is complicated and non-linear which is difficult to model with a single linear methodology. Therefore, the non-linear assessment methodology is needed. With the development of computer technology and artificial intelligence, the artificial neural network (ANN) has been used in the safety assessment in the urban rail transit system. The ANN has the characterizations of the parallel computation, nonlinear mapping in high level and self-organization structure. And the usage of ANN does not need the relationship between the input and output, without having a large number of parameters. The common ANN for assessing the operation safety of urban rail transit systems is back-propagation neural network (BPNN). However, the assessment using the BPNN needs a larger number of training samples since small samples will lead to local minimum values and result in inaccurate predicted values. In order to improve the accurate of the assessment, the generalized regression neural network (GRNN) could be used for the evaluation. The GRNN was developed based on the radial basis function neural network (RBFNN), which could obtain the stable, high accurate prediction results compared to the BPNN. Therefore, this paper presented an assessment methodology for evaluating the

\footnotetext{
* Corresponding author: gwei@live.com
} 
operation safety of urban rail transit system by using the GRNN. In addition, the BPNN was also used to compare with the GRNN.

\section{Methodology}

The ANN was one hot topic in the field of artificial intelligence. It establishes a simple model with the different networks consisted of a large number of nodes (neurons) to simulate the information processing of the human brain. Each node (neuron) represents a specific output called as the activation function. The connection between two nodes represents the weighted value for the signal, which can be seen as the memory of the ANN. The output of the ANN finally depends on the connection, the weighted value and the activation function. The common ANNs include the back-propagation neural network (BPNN), the radial basis function neural network (RBFNN) and the general regression neural network (GRNN).

\subsection{Back Propagation Neural Network}

The BPNN is one of the most popular and successful ANN. A typical BPNN consists of three layers called as the input layer, the hidden layer and the output layer (see Figure 1). The connections exit between the input layer and the hidden layer, and between the hidden layer and the output layer. The BPNN is a feed forward multiplelayer network. The signal is forward-propagation from the input layer to the output layer by passing through the hidden layer. And the error is back-propagation from the output layer to the input layer by passing through the hidden layer with adjusting the weight and the bias. The activation function in the hidden layer is generally the sigmoid function with the amplification of the non-linear effect which results in the nonlinear transformation output in the range of 0 and 1.

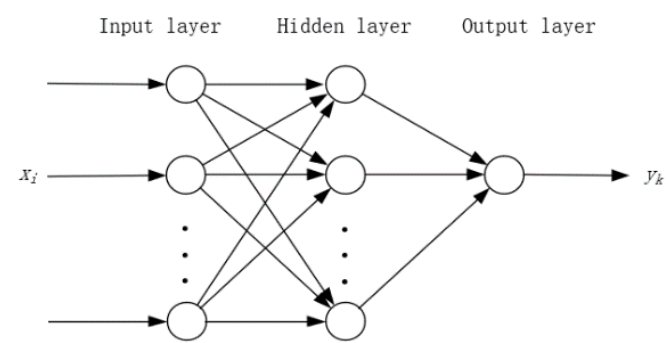

Fig. 1. Structure of the back-propagation neural network

\subsection{Generalized Regression Neural Network}

The GRNN was developed based on the RBFNN. The output of the GRNN uses the density function instead of the lease square multiplication of radial basis function in the RBFNN.

The equation of the output in the GRNN is:

$$
\widehat{Y}=\mathrm{E}(y \mid X)=\frac{\int_{-\infty}^{\infty} y f(X, y) d y}{\int_{-\infty}^{\infty} f(X, y) d y}
$$

where $\hat{Y}$ is the output of the ANN in the condition of $X$; $f(X, y)$ is the joint probability density function. The general joint probability density function is the Gaussian function.

Compared with the RBFNN, the training of the GRNN is more convenient and suitable for the curve fitting.

\subsection{Precisions of Assessment}

In order to evaluate the predictions of the ANNs, the error test formulas will be used in the assessment. The formulas are shown in Table 1 . The grades of the evaluations with parameters $\mathrm{c}$ and $\mathrm{p}$ were shown in Table 2.

Table 1 Error Test Formula

\begin{tabular}{|c|c|c|}
\hline & Residuals & Raw data \\
\hline $\begin{array}{c}\text { Mean } \\
\text { difference }\end{array}$ & $\bar{\varepsilon}=\frac{1}{n} \sum_{i=1}^{n} \varepsilon_{i}$ & $\bar{X}=\frac{1}{n} \sum_{i=1}^{n} X_{i}$ \\
\hline Variance & $S_{1}^{2}=\frac{1}{n} \sum_{i=1}^{n}\left(\varepsilon_{i}-\bar{\varepsilon}\right)$ & $S_{2}^{2}=\frac{1}{n} \sum_{i=1}^{n}\left(X_{i}-\bar{X}\right)$ \\
\hline $\begin{array}{c}\text { The ratio of } \\
\text { posterior } \\
\text { difference }\end{array}$ & \multicolumn{2}{|c|}{$c=S_{1} / S_{2}$} \\
\hline $\begin{array}{c}\text { Small error } \\
\text { probability }\end{array}$ & $p=p\left\{\left|\varepsilon_{i}-\bar{\varepsilon}\right|<0.6745 S_{2}\right\}$ \\
\hline
\end{tabular}

Table 2 Classifications of the Prediction

\begin{tabular}{|c|c|c|}
\hline $\begin{array}{c}\text { Precision of } \\
\text { predictions }\end{array}$ & $\mathrm{P}$ & $\mathrm{c}$ \\
\hline Excellent (1st level) & $>0.95$ & $<0.35$ \\
\hline Good (2nd level) & $>0.8$ & $<0.5$ \\
\hline Poor (3rd level) & $>0.7$ & $<0.65$ \\
\hline Fail (4th level) & $\leq 0.7$ & $\geq 0.65$ \\
\hline
\end{tabular}

\subsection{Data}

The operation safety of the urban rail transit system is a complex non-linear problem which includes many factors affecting the operation safety. According to the published literature, the typical factors were chosen from the four major categories: persons, machines, environment, and management. Those factors and the scores graded by the experts can be found in the reference [5]

\section{Result}

The prediction values by using the BPNN and GRNN with the experts' scores were shown in Table 3 and Figure 2. The classification of the predictions can be seen in Table 4. The results indicated that the predictions by using the GRNN ,the c value is 0.0557 , which is smaller than 0.35 ; and the $\mathrm{p}$ value is 1.0 , which is greater than 0.95 . 
Therefore, the precision of the predictions by using the GRNN could reach the level 1. While using the BPNN, the $\mathrm{c}$ value is 0.3510 and the $\mathrm{p}$ value is 1.0 . By checking the Table 2, the precision of the predictions could reach the level 2. The above results showed that both the GRNN and the BPNN can be used in the evaluation of operation safety of urban rail transit system. However, the GRNN can achieve high accurate evaluation.

Table 3 Predictions by using ANNs

\begin{tabular}{|l|l|l|l|l|l|}
\hline \multirow{2}{*}{ No. } & \multirow{2}{*}{$\begin{array}{c}\text { Expert } \\
\text { Score }\end{array}$} & $\begin{array}{c}\text { Evaluation } \\
\text { value }\end{array}$ & $\begin{array}{c}\text { Relative } \\
\text { error }\end{array}$ & $\begin{array}{c}\text { Evaluation } \\
\text { value }\end{array}$ & $\begin{array}{c}\text { Relative } \\
\text { error }\end{array}$ \\
\cline { 5 - 6 } & 28.32 & 20.2324 & $-28.56 \%$ & 25.3494 & $-10.49 \%$ \\
\hline 2 & 23.77 & -13.258 & $-155.78 \%$ & 27.5209 & $15.78 \%$ \\
\hline 3 & 20.49 & 14.094 & $-31.22 \%$ & 26.7587 & $30.59 \%$ \\
\hline 4 & 41.86 & 44.9082 & $7.28 \%$ & 48.9218 & $16.87 \%$ \\
\hline 5 & 76.38 & 80.0779 & $4.84 \%$ & 82.1324 & $7.53 \%$ \\
\hline 6 & 90.02 & 82.7729 & $-8.05 \%$ & 89.9155 & $-0.12 \%$ \\
\hline 7 & 85.98 & 97.8053 & $13.75 \%$ & 87.2624 & $1.49 \%$ \\
\hline 8 & 35.15 & 66.5877 & $89.44 \%$ & 44.0262 & $25.25 \%$ \\
\hline 9 & 73.09 & 67.4521 & $-7.71 \%$ & 77.4184 & $5.92 \%$ \\
\hline 10 & 45.32 & 46.7313 & $3.11 \%$ & 62.6217 & $38.18 \%$ \\
\hline 11 & 79.57 & 77.1529 & $-3.04 \%$ & 86.428 & $8.62 \%$ \\
\hline 12 & 83.99 & 79.0051 & $-5.94 \%$ & 87.4966 & $4.18 \%$ \\
\hline 13 & 85.55 & 75.8703 & $-11.31 \%$ & 88.1238 & $3.01 \%$ \\
\hline 14 & 87.67 & 82.9368 & $-5.40 \%$ & 87.3781 & $-0.33 \%$ \\
\hline 15 & 36.41 & 98.3514 & $170.12 \%$ & 41.3050 & $13.44 \%$ \\
\hline
\end{tabular}

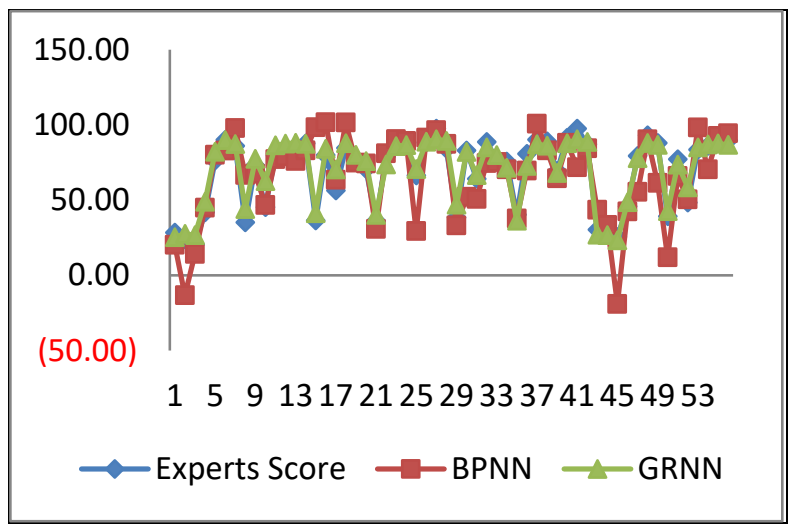

Fig. 2. Comparisons between the experts' scores and the prediction values by using the BPNN and GRNN

Table 4 Grades of the Predictions by using ANNs

\begin{tabular}{|c|c|c|c|c|c|}
\hline ANN & $\mathbf{S}_{\mathbf{1}}$ & $\mathbf{S}_{\mathbf{2}}$ & $\mathbf{C}$ & $\mathbf{P}$ & Grade \\
\hline BPNN & 283.6973 & 808.3613 & 0.3510 & $100 \%$ & Good \\
\hline GRNN & 26.1583 & 469.3022 & 0.0557 & $100 \%$ & Excellent \\
\hline
\end{tabular}

\section{Conculsion}

The paper presented a methodology by employing the GRNN to evaluate the operation safety of urban rail transit. The relative errors between the predictions and the experts' score are $5.38 \%$ and $32.04 \%$ for the GRNN and BPNN. The precision of predictions by using GRNN could reach the level 1 , which is very close to the experts' scores. Therefore, the GRNN is capable of evaluating the operation safety of urban rail transit very well. Though the relative error for the BPNN is about $32.04 \%$, the precision of the prediction could reach the level 2 (good) which indicated that the BPNN could also evaluate and predict the operation safety of the urban rail transit system.

\section{References}

1. K.R. Huang. A Simulation Study on Smoke Distribution and Evacuation of People in Subway Station Fire. Master Thesis. Beijing University of Technology. (2007)

2. London Underground Limited.. Quantified Risk Assessment Update 2001-Network \& Business Unit Summary. (2001)

3. London Underground Limited.. Railway Safety Profile of Safety Risk on Rail Track PLC-Controlled Infrastructure. (2001)

4. R.J. Adduci, A.Boyd, J.Caton. Handbookfor Transit Safety and Security Certification.

5. L.L. Jiang (2014). Evaluation of subway operation safety based on the GA-BP network. Doctoral Thesis. Dalian Jiaotong University. (2002) 